\title{
The Research of Industry Evaluation Index System of Training Quality for Software Engineering Professional Degree Postgraduate
}

\author{
Zhang Yao, Li Shu-yu, Li Ze-kun \\ School of Computer Science, Shaanxi Normal University, Xi'an, China
}

\section{Email address:}

zyao@snnu.edu.cn (Zhang Yao)

\section{To cite this article:}

Zhang Yao, Li Shu-yu, Li Ze-kun. The Research of Industry Evaluation Index System of Training Quality for Software Engineering Professional Degree Postgraduate. Science Innovation. Vol. 5, No. 2, 2017, pp. 122-126. doi: 10.11648/j.si.20170502.20

Received: November 9, 2016; Accepted: April 17, 2017; Published: April 20, 2017

Abstract: This article mainly proposes a relatively complete software engineering discipline postgraduate Education quality Evaluation system industry based on AHP, to solve the prevalence of a single body, the lack of software professional degree characteristics, lack of proper assessment methods of a series of problems. The established evaluation system is guided by the professional qualification certification, and in Shaanxi Normal University, college of computer science software engineering as an example, using the system to complete industry assessment and occupational qualification certification.

Keywords: Software Engineering Professional Degree, AHP, Evaluation Index System,

Professional Qualification Certification

\section{研究生培养质量的行业评估指标体系研究----- 以软件工程学科专业学位为例}

张瑶, 李蜀瑜, 李泽旎

计算机科学学院, 陕西师范大学, 西安, 中国

邮箱

zyao@snnu. edu. cn（张瑶）

摘要：软件工程学科的专业学位研究生培养质量评估体系普遍存在着评估主体单一、缺乏软件专业学位特色、缺乏完 善的评估方法和手段等一系列问题, 针对这些不足, 提出一种基于AHP构建的较为完整的软件工程学科专业学位研究生 培养质量行业评估指标体系。以职业资格认证为导向建立评估体系, 并以陕西师范大学计算机科学学院软件工程专业 为例, 进行行业评估和职业资格认证。

关键字: 软件工程专业学位, AHP, 评估指标体系, 职业资格认证

\section{1. 引言}

高校是培养人才，向社会和企业输送专业人才的地方。 近年来, 随着IT产业迅速发展, IT企业的快速增加, 软件
专业人才的大量需求, 促使高校对软件工程专业人才的培 养应更多的满足IT企业的要求。IT企业需要能够从事软件 分析、设计、开发、管理等相关工作的人才，但因为多数 高校对软件行业、职业特点的不了解和不重视, 导致软件 
工程专业学位研究生的培养缺乏针对性 [1]。这些现象同 样引起了国家职能部门的高度重视, 因此, 为了促使高校 的教育更加符合企业的实际需求, 国家出台了一系列政策, 如教育部启动了工程教育专业认证等 [2]。

当前, 多数高校在软件工程学科的专业学位研究生培 养过程中由于沿用了学术学位的质量评估体系, 使得该教 育普遍存在着 “同化、矮化、弱化” 的弊端 [3]。因此, 针对软件工程学科的专业学位研究生培养质量评估体系 普遍存在的评估主体单一、缺乏软件专业学位特色、缺乏 完善的评估方法和手段 [4]等一系列问题, 提出一种以资 格认证为导向, 基于AHP构建的较为完整的软件工程学科 专业学位研究生培养质量行业评估指标体系。

通过该评估体系进行行业评估和职业资格认证，可以 有效发现专业学位研究生培养过程中的问题, 剖析成因, 从而有针对性地实施管理及控制的措施, 提高软件工程学 科专业学位研究生的培养质量 [5]。因此, 研究与构建一 个较为完整的软件工程学科专业学位研究生培养质量的 外部行业评估体系, 具有十分重要的理论意义和较好的应 用价值。

\section{2. 软件工程学科专业学位研究生培养质量的行 业评估指标体系}

通过创建陕西师范大学软件工程学科专业学位研究 生培养质量的行业外部评估体系, 构建的评估体系IT企业 与行业协会等实践单位作为评估主体纳入整个软件工程 学科专业学位研究生教育体系中来, 一方面能够有效的改 变陕西师范大学软件工程学科专业学位研究生培养质量 的评估主体单一的问题, 另一方面在培养实践能力的过程 中, 还能承担部分培养成本。相信通过对实践中的数据进 一步的进行分析探讨后, 能够对软件工程学科专业学位研 究生培养质量的行业外部评估体系进一步完善。

完善的评估体系的建立, 是对软件工程学科专业学位 研究生教育本质进行更的进一步探索, 是对研究生专业学 位的整体系统进行的改革和创新, 是培养高层次、实用型、 复合型的软件工程人才的一项重要任务。因此, 软件工程 学科专业学位研究生培养质量的行业评估指标体系的建 立, 将以职业资格认证为导向, 以行业协会和IT企业为主 要评估者, 以AHP为评估方法, 对体系进行分析和构建。

\section{1. 软件工程学科专业学位研究生培养中存在的问题}

软件工程是研究用工程化方法去构建和维护有效、实 用和高质量的软件的一门学科 [6]。以往高校着力培养软 件工程学科的专业学位研究生的综合素质、专业知识、实 践能力, 使学生能够从事简单的软件分析、设计、开发、 管理等相关工作 $[7]$ 。但随着国家对专业学位研究生教育 培养目标和定位要求的转变, 及国家在科研评估体系的重 大改革, 促使软件工程学科的专业学位研究生的培养质量 评估与IT企业深度结合, 为IT企业提供更多高质量的人才 $[8]$ 。

就目前情况而言, 大多数高校在软件工程学科的评定 依旧使用学术学位的质量考核标准, 并不能很好的考虑到
软件工程学科专业学位本身的特点, 使得该教育普遍存在 着 “同化、矮化、弱化” 的弊端 [3]。对陕西师范大学进 行调研发现, 该校软件工程学科专业学位研究生评估体系 主要存在以下三个方面的问题:（1）评估主体过于单一, 评估体本身只能通过学校的专业课以及毕业论文成绩进 行评价, 无法很好的与社会用人企业的人才需求对接; (2) 缺乏软件专业学位特色, 实践本身不能很好的与社会的技 术需求相结合; (3) 缺乏完善的评估方法和手段, 没有 完善的框架对整个高校培养质量进行评估 [4]。

\section{2. 软件工程学科专业学位研究生培养目标}

软件工程专业学位研究生教育具有鲜明的职业教育 属性, 以专业实践为导向, 侧重于实践与应用相结合, 为 IT行业培养需要的高层次人才。从培养方式看, 软件工程 专业学位研究生教育更注重对职业实践能力的培养, 具有 自己独特的培养目标、培养模式和人才质量标准。因此, 软件工程专业学位研究生教育应是以职业应用为导向, 侧 重于实践和应用能力的提升 [9], “高层次”、“应用型”、

“专业人才” 应是其培养目标的重点与核心。

陕西师范大学软件工程学科的专业学位研究生培养 的目标是: 服务于经济建设, 理工结合, 基础扎实, 素质全 面, 实践能力和创新能力突出, 能够从事软件的分析、设 计、开发、管理和服务等相关工作。也就是说, 培养的软 件工程专业人才应该具备实用性、复合型的特征。

\section{3. 软件工程学科专业学位研究生培养原则}

软件工程专业学位研究生培养模式是一个复杂的体 系, 它所构成的有机整体之间的元素是相互联系, 相互影 响的。因此, 在构建一个合理、科学的专业学位研究生培 养质量的评估指标体系过程中, 必须紧紧的把握以下三点 原则:

（1）科学性原则: 应以综合评价为主, 多采用分析 评价指标, 使定量指标和定性指标有效结合;

（2）系统性原则：每个评价指标既要从整体上对专 业学位研究生培养过程进行把握, 还要避免以点带面, 以 及指标之间的重叠问题;

(3) 可操作性原则: 专业学位研究生培养质量的评 估体系指标都需要通过实践来进行验证, 为了提高整个体 系的可操作性, 可以考虑在保证对整体的研究生培养过程 没有太大影响的情况下, 尽可能的简化评价指标 [10]。

\section{4. 基于AHP软件工程学科专业学位研究生培养质量的行 业评估指标体系}

针对软件工程专业学位研究生教育质量评估体系存 在的问题, 需要对评估体系上实现转变, 即把专业学位研 究生培养质量的评估从单一的高校评估, 转变为政府主导 下的外部行业和社会评估为主、高校评估为辅的多元评估, 意味着培养质量评估不再是单一的课程成绩和论文评估, 更多的是职业资格、科研创新、社会服务评估等。

由于高等教育本身具有滞后性, 引入高等教育外部利 益关系人（特别是软件行业协会和IT企业）参与评估, 就 显得非常有必要, 这样才能推动高校及时了解社会需求, 
合理改进培养方案, 促进软件工程学科的专业学位研究生 适应未来社会。

在评估指标体系中, 职业资格认证指标将是一个非常 重要的指标。该指标要求毕业生具备IT企业要求的知识、 能力和职业素养, 并具有基本的从业条件。在软件工程专 业学位研究生培养过程中引入资格认证, 可以依据职业资 格认证的相关要求, 整合师资队伍、调整课程体系、增加 实训平台, 将职业资格认证融合到高校课程体系及教育教 学活动中, 带动高校的教育教学改革和学科建设 [11]。以 学校为中心选取的评估指标往往不能体现IT企业对软件 工程专业学位研究生的影响程度。高校需要了解IT企业真 正的需求, 在培养过程中增加与IT企业的链接, 培养学生 实践能力。因此，建立的评估指标体系中包括高校、行业 协会和IT企业等社会用人单位三个方面, 其中, 行业协会 和IT企业将是最重要的评估者。

层次分析法 (简称AHP) 是一种对定性问题进行定量分 析的方法 [12]。在评估指标体系中, 拟采用选择AHP作为 方法, 依据行业协会和IT企业提出的意见对评估指标进行 综合分析后, 判断评估体系中指标的重要度, 然后进行两 两比较建立判断矩阵, 利用矩阵的特征向量求解各指标的 权重值, 最后得出对于总指标的权重, 并对教育质量实现 总体综合评估 [13]。

培养的软件工程学科的专业学位研究生应具有良好 的综合素质和职业道德、扎实的专业基础知识, 并且具有 良好的软件设计与实现、项目管理、沟通和合作、前沿学 习和创新能力 $[14]$ 。经调查问卷及行业协会和IT行业专家 的意见, 拟考虑三大类评估指标: 思想道德指标类、专业 知识指标类、职业素质指标类。

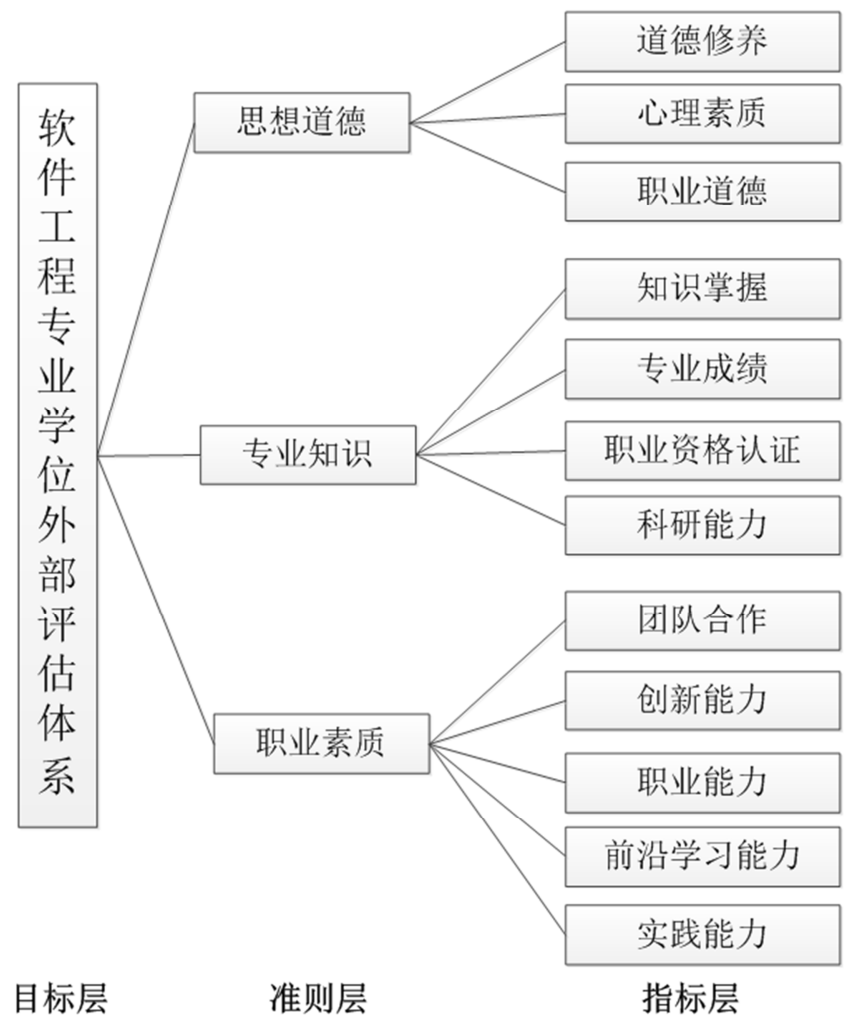

图1 软件工程学科专业学位研究生培养质量的行业评估指标体系。
如图1所示, 思想道德指标类包含道德修养、心理素 质、职业道德等指标; 专业知识指标类包含知识掌握、专 业成绩、职业资格认证、科研能力等指标; 职业素质指标 类包含团队合作、创新能力、职业能力、前沿学习能力、 实践能力等指标。其中专业知识指标类和职业素质指标类 将是重点指标类。评估者均可根据学校实际情况对上述指 标再分层和细化。

\section{3. 基于AHP陕西师范大学软件工程学科专业学位 研究生培养质量评估指标体系}

按照建立的评估体系, 具体指标描述如表 1 所示, 以 陕西师范大学计算机科学学院软件工程专业为例, 行业协 会和IT企业对专业学位研究生进行行业评估和职业资格 认证。运用AHP对体系进行构建, 根据评估体系中指标的重 要度, 进行两两比较建立判断矩阵, 利用矩阵的特征向量 求解各指标的权重值, 并且需要对判断矩阵进行一致性检 验, 来确定其有效性。

在AHP中, 针对指标因子无法直接定量的情况, 进行 定性分析, 根据重要程度, 采用1-9比例标度对重要性程 度赋值。经调查问卷及行业协会和IT行业专家的意见对指 标进行赋值, 并根据指标之间相对重要性的比较得到一个 判断矩阵, 利用数学公式求出判断矩阵的最大特征根 $\lambda$ 及 相应的特征向量W' , 对特征向量归一化后作为指标的权 重向量W。如表2-5所示, 详细介绍各级指标重要程度及所 占权重。由于这种计算相对权重向量的方法, 当判断矩阵 过多偏离一致性时, 其可信度就值得怀疑。因此利用最大 特征值计算一致性指标CI并查找相应的平均随机一致性 指标RI $[15]$, 计算一致性比例 $\mathrm{CR}$ 。其中: $\mathrm{CI}=\frac{\lambda-n}{n-1}, \mathrm{CR}=\frac{C I}{R I}$ 。 通常认为 $C R<0.1$ 时, 判断矩阵具有满意的一致性, CR越小, 判断矩阵的一致性越好。在评估过程中, 运用数学软件对 建立的判断矩阵逐个进行一致性检验, 通过检验即可利用 MATLAB计算权重, 未通过一致性检验的则需再进行讨论并 重新构建。

表1 陕西师范大学专业学位研究生培养质量评估指标体系。

\begin{tabular}{lll}
\hline 一级指标 & 二级指标 & 说明 \\
\hline & 道德修养 $\mathrm{A} 1$ & 在实习单位中的意识和行为 \\
思想道德 $\mathrm{A}$ & 心理素质 $\mathrm{A} 2$ & 在实习单位承受压力的能力 \\
& 职业道德 $\mathrm{A} 3$ & 是否遵守IT行业的基本道德准则 \\
& 知识掌握B1 & 实习职位与在校期间专业知识的 \\
& 学习的关联度 \\
专业知识B & 专成绩 $\mathrm{B} 2$ & 在校专业课成绩 \\
& 职业资格认证B3 & 考取的资格认证证书等 \\
& 科研能力 $\mathrm{B} 4$ & 在校期间申请的专利、软件著作 \\
& 权、科技发明等 \\
& 团队合作 $\mathrm{C} 1$ & 在实习单位的项目中体现的团队 \\
& 之间良好的交流与组织协调能力 \\
& 创新能力 $\mathrm{C} 2$ & 在实习单位中用新的思想、方法解 \\
& 决问䞨的能力 \\
& 职业能力 $\mathrm{C} 3$ & 软件设计与实现能力、良好的项目 \\
& 管理能力 \\
& 前沿学习能力C4 & 对 IT技术发展的了解及参与学习 \\
& 的能力 \\
& 实践能力 $\mathrm{C} 5$ & 在实习单位中对专业知识的运用 \\
& & 程度 \\
\hline
\end{tabular}


表2 一级指标的判断矩阵 $\mathrm{S}$ 。

\begin{tabular}{lllll}
\hline 一级指标 $\mathrm{S}$ & $\mathrm{A}$ & $\mathrm{B}$ & $\mathrm{C}$ & 权重 \\
\hline $\mathrm{A}$ & 1 & $1 / 2$ & $1 / 4$ & 0.1429 \\
$\mathrm{~B}$ & 2 & 1 & $1 / 2$ & 0.2857 \\
$\mathrm{C}$ & 4 & 2 & 1 & 0.5714 \\
\hline
\end{tabular}

表3 二级指标 $\mathrm{A}$ 的判断矩阵 $\mathrm{A}$ 。

\begin{tabular}{lllll}
\hline 二级指标A & $\mathbf{A 1}$ & $\mathbf{A 2}$ & $\mathbf{A 3}$ & 权重 \\
\hline $\mathrm{A} 1$ & 1 & $1 / 2$ & 1 & 0.2599 \\
$\mathrm{~A} 2$ & 2 & 1 & 1 & 0.4126 \\
$\mathrm{~A} 3$ & 1 & 1 & 1 & 0.3275 \\
\hline
\end{tabular}

表4 二级指标 $\mathrm{B}$ 的判断矩阵 $\mathrm{B}$ 。

\begin{tabular}{llllll}
\hline 二级指标B & B1 & B2 & B3 & B4 & 权重 \\
\hline B1 & 1 & 3 & 2 & 2 & 0.4258 \\
B2 & $1 / 3$ & 1 & $1 / 2$ & 1 & 0.1484 \\
B3 & $1 / 2$ & 2 & 1 & 1 & 0.2312 \\
B4 & $1 / 2$ & 1 & 1 & 1 & 0.1945 \\
\hline
\end{tabular}

表 5 二级指标 $\mathrm{C}$ 的判断矩阵 $\mathrm{C}$ 。

\begin{tabular}{lllllll}
\hline 二级指标C & C1 & C2 & C3 & C4 & C5 & 权重 \\
\hline C1 & 1 & $1 / 2$ & $1 / 4$ & 2 & $1 / 2$ & 0.1052 \\
C2 & 2 & 1 & $1 / 2$ & 5 & 2 & 0.2558 \\
C3 & 4 & 2 & 1 & 7 & 2 & 0.4102 \\
C4 & $1 / 2$ & $1 / 5$ & $1 / 7$ & 1 & $1 / 3$ & 0.0544 \\
C5 & 2 & $1 / 2$ & $1 / 2$ & 3 & 1 & 0.1744 \\
\hline
\end{tabular}

表6 陕西师范大学软件工程学科专业学位研究生培养质量评估指标体 系。

\begin{tabular}{lllll}
\hline & $\begin{array}{l}\text { 思 想 道 } \\
\text { 德 }\end{array}$ & $\begin{array}{l}\text { 人才培 } \\
\text { 养B }\end{array}$ & $\begin{array}{l}\text { 社会评估 } \\
\mathbf{C}\end{array}$ & $\begin{array}{l}\text { 综 合 权 重 } \\
\text { 值 }\end{array}$ \\
\cline { 2 - 5 } & $\mathbf{0 . 1 4 2 9}$ & $\mathbf{0 . 2 8 5 7}$ & $\mathbf{0 . 5 7 1 4}$ & $\mathbf{1}$ \\
\hline 道德修养A1 & 0.2599 & & & 0.0371 \\
心理素质A2 & 0.4126 & & & 0.0590 \\
职业道德A3 & 0.3275 & & & 0.0468 \\
知识掌握B1 & & 0.4258 & & 0.1217 \\
专业成绩B2 & & 0.1484 & & 0.0424 \\
职业资格认证B3 & & 0.2312 & & 0.0661 \\
科研能力B4 & & 0.1945 & & 0.0556 \\
团队合作C1 & & & 0.1052 & 0.0601 \\
创新能力C2 & & & 0.2558 & 0.1461 \\
职业能力C3 & & & 0.4102 & 0.2344 \\
前沿学习能力C4 & & & 0.0544 & 0.0311 \\
实践能力C5 & & & 0.1744 & 0.0996 \\
\hline
\end{tabular}

表6描述陕西师范大学软件工程学科专业学位研究生 培养质量各评估指标所占综合权重, 从表中可以看出, 职 业能力占据较高权重, 我校在以后的研究生培养过程中, 应当加强软件工程专业学位研究生教育与职业能力的衔 接, 将行业协会和IT企业等相关单位作为实践培养和评估 主体纳入专业学位研究生教育, 并且充分利用校内外实训 平台和产学研基地, 提高学生解决实际问题的能力。

创新能力、专业知识、合作能力同样占据较高的权重。 为了培养学生创新能力, 我校应同IT企业合作组建学生创 新创业中心, 并且提供资金和资源资助。学校应更多的考 虑企业需求设置专业课程, 对教学内容进行整合, 提高学 生的知识掌握能力。

\section{4. 结论}

建立一个科学、有效的、可操作性强的评估指标体系 是为了更好地建立软件工程学科专业学位研究生培养方 案, 提高培养质量。本文以陕西师范大学为例, 介绍体系 内容及其建立过程, 并对软件工程专业学位研究生进行行 业评估和职业资格认证, 将IT企业与行业协会等实践单位 作为评估主体纳入整个软件工程学科专业学位研究生教 育体系中来, 从而为高层次软件工程人才培养提供了理论 依据。利用AHP法对软件工程学科专业学位研究生评估指 标体系进行的构建, 仍需在实践中不断加以完善, 并探索 出更符合实际的评估体系。

\section{致谢}

1. 2014年教育部校企合作专业综合改革项目 “大学计 算机核心课程教学改革与课程建设”，2014-2015

2. 2016年陕西师范大学校级重点教学改革综合研究 项目 “面向师范院校计算机专业本科生的工程能力培养与 评估研究”，2016-2017

\section{参考文献}

[1] 李占宣. 对地方高校软件工程应用型人才培养的思考 [J]. 教育探索, 2014, 34(8)：87-88。

[2] 孙雅因. 合作学习方式在高校体育舞蹈教学中实验研究 [D]. 辽宁师范大学, 2011。

[3] 刘六生, 冯用军. 高等教育研究中的数学方法 $[\mathrm{M}]$. 北京: 科 学出版社, 2009。

[4] 周晓婧, 杨蜀康. 基于AHP的专业学位研究生教育外部质量 评价体系研究 [J]. 浙江理工大学学报: 社会科学 版, 2014, 32 (3) : 234-237。

[5] 李娟, 孙雪, 王守清. 专业学位研究生教育的外部质量评价 体系的构建：以职业资格认证为导向 [J]. 黑龙江高教研 究, 2010, 199 (11) :57-59。

[6] 朱涛. 基于软件工程理论的中小企业视觉品牌管理的现实 性初探 [J]. 大众文艺, 2010, 32 (12) :218-219。

[7] 庞洪江, 陈欢. 关于软件工程专业硕士研究生培养的新思考 [J]. 教育探索, 2013, 33(10): 54-55。

[8] 张宁. 从创新驱动发展战略看高校软件工程专业发展 [J]. 时代教育, 2014, 11(2)：153-154。

[9] 梁丽萍, 段荣娟. 我国专业学位研究生教育现状与思考 [J]. 山西高等学校社会科学学报, 2015, 27 (5) :88-92。

[10] 郑浩, 王欣. 全日制专业学位研究生培养模式评价指标体系 的构建 [J]. 价值工程, 2013, 32 (11) : 249-250。

[11] 高鸿. 改革专业学位研究生培养模式完善高层次应用型人 才培养体系 [J].中国职业技术教育. 2014, 22 (21): 94-97。 
[12] 王莲芬, 许树柏. 层次分析法引论 [M]. 北京: 中国人民大学 出版社. 1990。

[13] 陈全成, 陈东清. 经管类研究生综合素质评价研究: 基于层 次分析法 [J]. 长春理工大 学学报, 2011, 24 (7) : 132-134。
[14] 陈东明, 杨广明, 李丹程, 谭振华. 软件工程硕士研究生培养 方案改革 $[J]$. 计算机教育, 2015, 13(3) : 1-6。

[15] 郭亚军. 综合评价理论、方法及应用 [M]. 北京: 科学出版 社, 200 。 